\title{
Design of Experiments for Factor Hierarchization in Complex Structure Modelling
}

\author{
Chaouki Kasmi $^{1,2 *}$, Marc Hélier ${ }^{2}$, Muriel Darces ${ }^{2}$ and Emmanuel Prouff ${ }^{1}$ \\ ${ }^{1}$ French Network and Information Security Agency - ANSSI, Paris, France \\ ${ }^{2}$ UPMC Univ. Paris 06, UR2, L2E, BC 252, 4 place Jussieu, 75005 Paris, France \\ *corresponding author, E-mail: chaouki.kasmi@ssi.gouv.fr
}

\begin{abstract}
Modelling the power-grid network is of fundamental interest to analyse the conducted propagation of unintentional and intentional electromagnetic interferences. The propagation is indeed highly influenced by the channel behaviour. In this paper, we investigate the effects of appliances and the position of conductors in a low voltage network. First, the power-grid architecture is described. Then, the principle of Experimental Design is recalled. Next, the methodology is applied to power-grid modelling. Finally, we propose an analysis of the statistical moments of the experimental design results. Several outcomes are provided to describe the effects induced by parameter variability on the conducted propagation of spurious compromising emanations.
\end{abstract}

\section{Introduction}

Many efforts are devoted to model the propagation of electromagnetic waves and their effects on complex structures [1-2] such as the low voltage distribution network [3-5]. Frequently, several input parameters of those models are not precisely defined. Moreover, the uncertainties on their value may impact the accuracy of the results. Hence, stochastic simulations, such as the Monte-Carlo approach [6-7], are generally applied to generate a large number of random configurations in which the effect of each parameter variability is estimated. In this paper, we propose to hierarchize the input variables depending on their impact on the propagation of electromagnetic interferences [8-10] in a modelled low voltage network. Thanks to the Experimental Design [11] calculation, we will be also able to estimate the interactions between those parameters.

In section 2 , the methodology used to model a low voltage distribution network is explained. In section 3, the principle of Experimental Design is recalled. The main lines of the formalism are introduced. Then, the methodology is applied in order to compute the effects induced by the variability of the input modelling parameters on a physical quantity (called observable). Finally, an analysis of the statistical moments of the experimental design results is proposed.

Further results are given to describe the effects of parameter variability on the conducted propagation of spurious compromising emanations generated by a computer connected to the distribution network.

\section{Low voltage network modelling}

The low voltage distribution network can be characterized by several parameters. The power-grid in France is a $230 \mathrm{~V}$ power-supply at $50 \mathrm{~Hz}$. Many conductor cross-sections, from 0.6 to $300 \mathrm{~mm}^{2}$, can be used depending on the maximum admissible current level. Moreover, different types of topologies (stars and chained topologies) can be encountered; a complex, large power-grid can be represented by the combination of elementary sub-networks characterised by: a known topology and a uniform cable cross-section. Finally, for safety requirements, several protections, such as fuses and breakers, may also be inserted. Nevertheless their non-linear behaviour will not be considered in this paper. In supplement of protecting devices, earthing schemes have been designed to enhance the safety principles. In the latter study, we mainly focus on the "Terra-Terra" earthing scheme based on the IEC-60364 standard. It means that the protective earth conductor is connected to the neutral one and to the metallic mass of the appliances.

\subsection{Cable modelling}

The low voltage network, depicted in Fig. 1, is based on a three copper wire cable, commonly named Phase (P), Neutral $(\mathrm{N})$ and Protective Earth (PE). In our analysis, it is assumed that the copper wires have a $1.5 \mathrm{~mm}^{2}$ cross-section and a $3 \mathrm{~m}$ length. Generally, the conductors are placed inside a PVC electrical raceway in order to protect them. In this study, its mean height above ground is $5 \mathrm{~cm}$ and its diameter is $3.5 \mathrm{~cm}$.

It can be seen that three female power sockets are available to connect the appliances to the power supply. The propagation of spurious compromising emanations induced by a computer connected to the power-grid, modelled by a voltage source $\mathrm{V}_{\text {Source }}$ equal to $1 \mathrm{~V}$ and its internal impedance $Z_{\text {Source }}(f)$, has been calculated.

In order to simulate a realistic connection of the test network to a larger power-grid, the measurement of the load impedance $Z_{\text {Power-grid, }}$ at the output observation point, has been performed. 


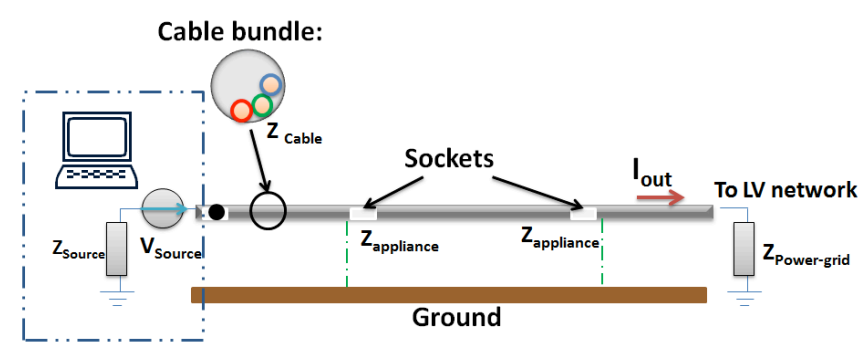

Figure 1: Modelled low voltage test network

Each cable bundle is characterise by an impedance matrix $\left[Z_{\text {Cable }}\right]$, function of the diameter of the conductors, their height above ground, their relative distances in the electric raceway and the frequency $f$. The connected electronic devices (printer, computer...) are taken into account via their input impedance matrix $\left[Z_{\text {Appliance }}\right]$. It can be assumed in this study that the magnitude of the common-mode endcurrent $\left|I_{\text {out }}\right|$, computed at the $Z_{\text {Power-grid }}$ level, is a function of the following parameters:

$$
\begin{aligned}
& \left|I_{\text {out }}\right|= \\
& \quad \alpha\left(V_{\text {Source }}, Z_{\text {Source, }}\left[Z_{\text {Cable }}\right],\left[Z_{\text {Appliance }}\right], Z_{\text {Power-grid }} f\right)
\end{aligned}
$$

The Multiconductor Transmission Line Theory [12] can be applied, assuming that the height of the conductors above the ground is smaller than the shortest wavelength. The conductor dielectric insulator losses are considered negligible in this study. Based on the above physical considerations and since the ground is considered a good conductor, it can be assumed that the propagated mode is a quasi-TEM one.

The conductors are randomly arranged in the raceway by defining the minimum admissible distance between wires. By using a plug-in of the CRIPTE code [13, 14], developed by the French Aerospace Laboratory (ONERA), named "Laplace calcul", 200 random cross-sections have been generated in order to build a significant realistic database. Three of them are depicted in Fig. 2. The RLGC parameters have been computed for each cross-section. The skin effect has been integrated within the per-unit length resistance parameter $R$ for copper wire, having a conductivity of $5.610^{7} \mathrm{~S} / \mathrm{m}$ by using Schelkunoff's formulation.
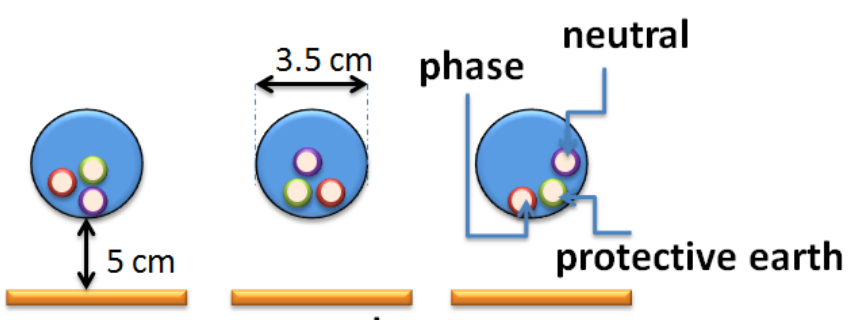

\section{ground}

Figure 2: Random cable cross-sections (limited to three cross-sections for graph readability)

\subsection{Measurements of the input impedance of appliances}

Propagation of high frequency signals in a low voltage network is highly impacted by the connected loads [15-16]. In order to simulate a more realistic network, it is mandatory to have a set of characterized appliances available.

By using a de-embedding technique [17] the measurement of the common mode input impedance of electric and electronic devices has been performed from $1 \mathrm{MHz}$ to $100 \mathrm{MHz}$. Seven classical appliances, representative of those usually found in an office, have been characterized such as a computer, a printer, a light and a phone.

Thanks to the statistical method presented in [17], ten simulated loads from each measured one have been derived by adding realistic variations to the measurements (reflection coefficient $S_{11}$ ). It is assumed that the obtained dispersion has a mean and a standard deviation derived as follows: the phase dispersion is chosen as $\pm 5^{\circ}$ and the amplitude level dispersion as $\pm 10 \%$ (in $\mathrm{dB}$ ). Moreover, the open-circuit socket has been introduced in the database in order to simulate an unloaded socket. The measured and simulated loads have been integrated into the CRIPTE code. In the latter study, it is considered that the database is finally composed of 71 loads.

\subsection{The CRIPTE Code}

One aspect of the Electromagnetic Topology theory [13, 14] consists in the decomposition of a non-uniform complex network into a set of connected uniform sub-networks by means of tubes and junctions [18]. The transmission lines are the tubes and the connecting points are the junctions as well as the input and output points. An iterative application of the electromagnetic topology theory allows the compaction of complex networks; an example is depicted in Fig. 3.

In order to model the interleaving of conductors in the raceway, each tube $\mathrm{T}_{i}$ ( $i$ denotes the $i$-th tube) is the compaction of sub-networks composed of four tubes $\mathrm{T}_{i j}$ of equal length characterised with the per-unit parameters obtained from random cable cross-section.

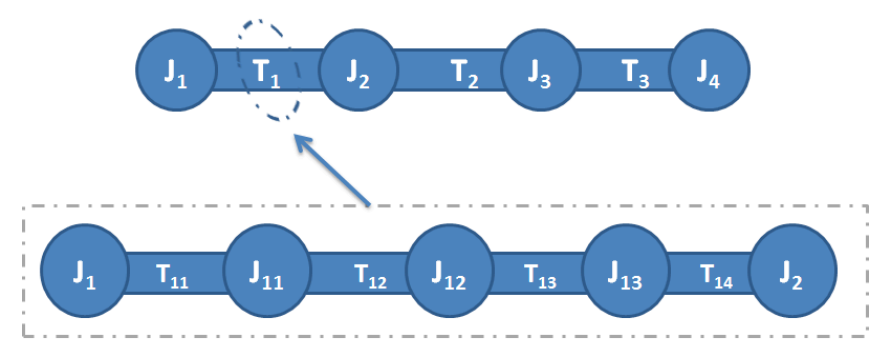

Figure 3: Topological description of the low voltage network under study

Using the CRIPTE code, the cable modelling methodology has been applied and the set of characterized loads has been 
used in order to compute the propagation of electromagnetic interferences in a realistic network. This leads us to characterise the tubes by the computed per unit length parameters of the three wire cable cross-sections and to describe the junctions by the measured input impedance of appliances.

\section{Generalized Experimental Design}

The Generalized Design of Experiments was first applied by Fisher [19] in agricultural engineering for growth rate analysis; it allows isolating the influence of each input variable involved in the model, called factor, on an output quantity, called observable; [11] is a good reference to start with this methodology.

In the following study, we apply this formalism in order to highlight the effects induced by each input parameter variability on a defined observable and to estimate their interactions. In this paper, we define the output observables as the mean $\mu$ and standard deviation $\sigma$ of the common mode end-current magnitude induced by a noisy computer (cf. Section 2); the end-current is defined as the sum of the currents in the three conductors in the electrical raceway at the end of the network. We supplement the analysis by showing the influence of the working frequency band on the parameter effects and their interactions; this has been investigated by the description of the probability density functions of the effects and their interactions. For this, an unbiased estimator of the statistical moments has been used.

\subsection{Principles}

The factors, which are controlled parameters, may take discrete or continuous values and also binary values. Usually these values are chosen in a set of levels. In order to process an experiment, it is assumed that there are several types of input parameters: the constants (diameter of wires, topology and length of the network) are fixed parameters precisely defined; random variables denote the parameters having their variability following a statistical distribution law that requires a Monte-Carlo simulation and factors. The principle is depicted in Fig. 4.

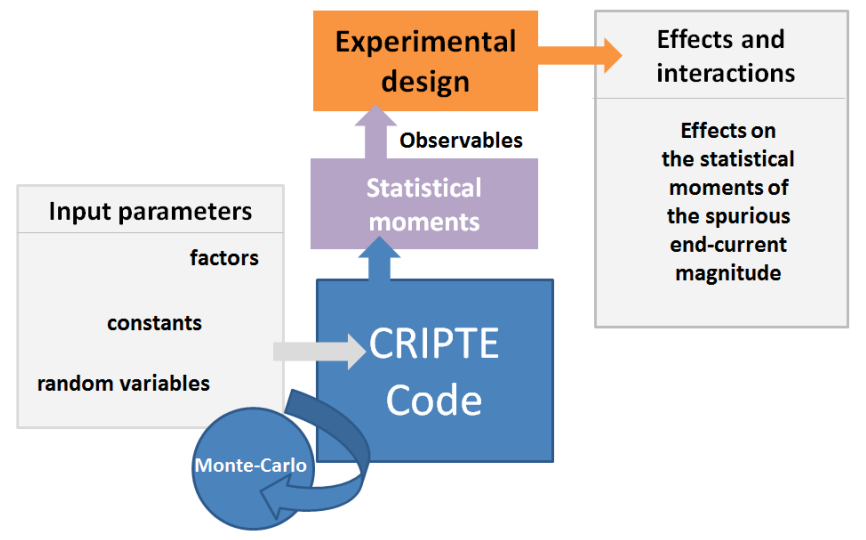

Figure 4: Description of the experimentation process
In our study, we have two binary factors $x_{1}$ and $x_{2}$ which are respectively the state of uniformity $\left(x_{1}=x_{I L}=0\right)$ or randomness $\left(x_{1}=x_{1 H}=1\right)$ of the wire arrangement and the connection state of the appliances (disconnected $\left(x_{2}=x_{2 L}=0\right)$ or connected $\left.\left(x_{2}=x_{2 H}=1\right)\right)$. The levels $x_{1 L}$, $x_{2 L}, x_{1 H}$ and $x_{2 H}$ are represented in Fig. 5.

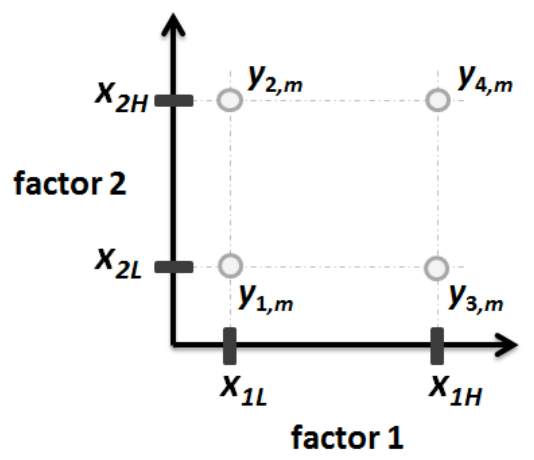

Figure 5: Points of experiments for a two-factor generalized experimental design

The corresponding observables are denoted $y_{1, m}$ to $y_{4, m}$ which are either the mean or the standard deviation of the endcurrent magnitude for the $i^{\text {th }}$ Monte Carlo (MC) simulation with the following considerations:

- $\quad y_{1, m}$ : uniform unloaded networks $\left(x_{1 L}, x_{2 L}\right)$;

- $\quad y_{2, m}$ : uniform loaded networks $\left(x_{I L}, x_{2 H}\right)$;

- $\quad y_{3, m}$ : non-uniform unloaded configurations $\left(x_{1 H}, x_{2 L}\right)$;

- $\quad y_{4, m}$ : non-uniform loaded networks $\left(x_{1 H}, x_{2 H}\right)$.

The parameter $m$ indicates the statistical moment under study of the end-current magnitude respectively $m=\mu$ for its mean and $m=\sigma$ for its standard deviation.

The combination of the resulting four points of experiments $\left(y_{1, m}\right.$ to $\left.y_{4, m}\right)$ allows the computation of both the effects induced by the factors and their interactions. They are defined at the working frequency $f$ as follows:

$$
\begin{aligned}
& a_{0, m}=1 / 4\left(y_{1, m}+y_{2, m}+y_{3, m}+y_{4, m}\right) \\
& a_{1, m}=1 / 4\left(-y_{1, m}+y_{2, m}-y_{3, m}+y_{4, m}\right) \\
& a_{2, m}=1 / 4\left(-y_{1, m}-y_{2, m}+y_{3, m}+y_{4, m}\right) \\
& a_{12, m}=1 / 4\left(y_{1, m}-y_{2, m}-y_{3, m}+y_{4, m}\right)
\end{aligned}
$$

The parameter $a_{0, m}$ is the mean level of the defined observable, $a_{1, m}$ and $a_{2, m}$ denote the effects induced by the two factors and $a_{12, m}$ is the interaction between both variables under study. In eq. (2), for readability, the frequency dependence of the observables and the effects has been omitted.

\subsection{Application to low voltage network modelling}

The Monte-Carlo simulations have been applied to derive random configurations following the defined considerations. 
200 cables cross-section have been generated. As a result, in our analysis 200 uniform networks can be simulated. Moreover, the model of the network is based on three one meter long tubes, thus $\mathrm{C}_{3}^{200}=1.310^{6}$ random configurations can be generated ( $\mathrm{C}$ refers to the binomial theorem).

Concerning the factor 2 (connection state of the appliances), networks have been analysed with the following considerations: loaded (the modelled network is defined with two sockets and the database of impedances is composed of 71 appliances, thus $\mathrm{C}_{2}^{71}==2.410^{3}$ random configurations can be generated) and unloaded $\left(x_{2 L}\right)$ by appliances.

It has been verified that the statistical moments (mean and standard deviation) are converging (less than $5 \%$ of variability) respectively for a number $N$ of experiment given as follows: $N\left(y_{1}\right)=200, \quad N\left(y_{2}\right)=10^{4}, \quad N\left(y_{3}\right)=10^{4}$ and $N\left(y_{4}\right)=10^{5}$.

In case of critical applications, the security of sensitive information is based on risk management. Thus, it is mandatory to estimate the maximum confidence of the Generalized Experimental Design results. The re-run of the Experimental Design applied to three sets of Monte-Carlo simulations (one of the three sets of simulation are depicted in Fig. 6 and Fig. 7) resulted in less than $2 \%$ of variation for the defined observables $\mu_{i}\left(\left|I_{\text {out }}\right|\right)$ and $\sigma_{i}\left(\left|I_{\text {out }}\right|\right)$.

\subsubsection{Mean effect, effects and interactions}

We investigate the potential interest of this formalism in order to study the propagation of electromagnetic interferences in the power-grid. For each frequency, a Design of Experiments is processed. The first statistical moments (mean) of the spurious end-current magnitude for each MC simulation are depicted in Fig. 6.

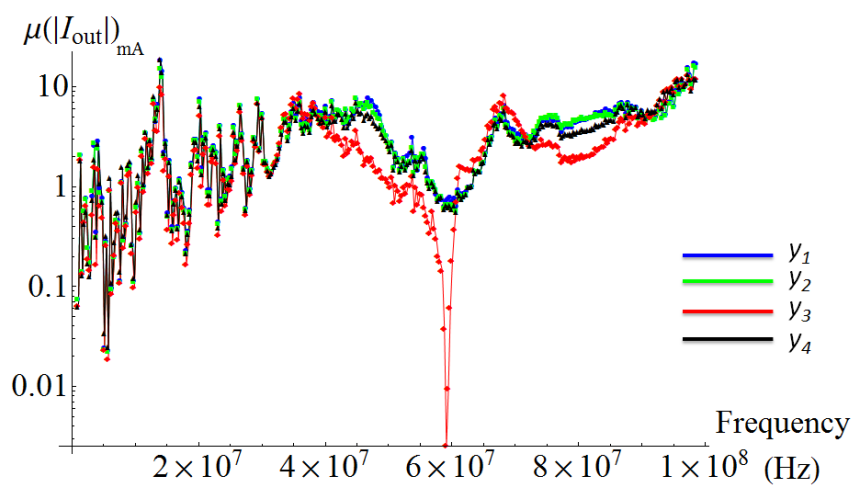

Figure 6: Computation of the end-current mean magnitude for the four configurations of the $\mathrm{MC}$ simulations

In order to get further details about how scores are concentrated around their mean for each frequency, the estimation of the standard deviation is performed for the four MC simulations. The resulting second-order moment are given in Fig. 7.

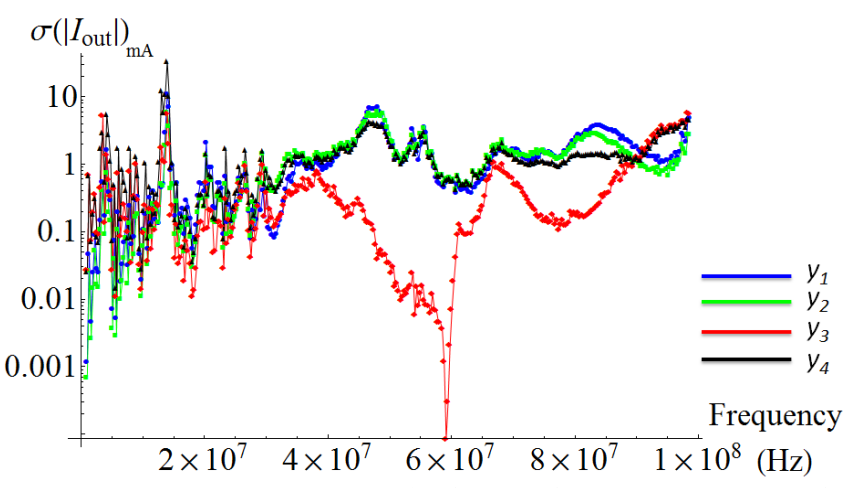

Figure 7: Computation of the end-current magnitude standard deviation for the four configurations of the $\mathrm{MC}$ simulations

It can be clearly noticed that the state of the appliances (connected or not) have a significant influence above $40 \mathrm{MHz}$ as depicted in Fig. 6 and Fig. 7.

By using expressions (2), the following parameters have been computed: the end-current mean magnitude $a_{0, \mu}$ (depicted in Fig. 8), the effects induced by the randomness position of wires in the network $a_{1, \mu}$ (depicted in Fig. 9) and by the connection state of appliances $a_{2, \mu}$ (depicted in Fig. 10) on the spurious end-current mean magnitude, and the interaction $a_{12, \mu}$ (depicted in Fig. 11) between both factors.

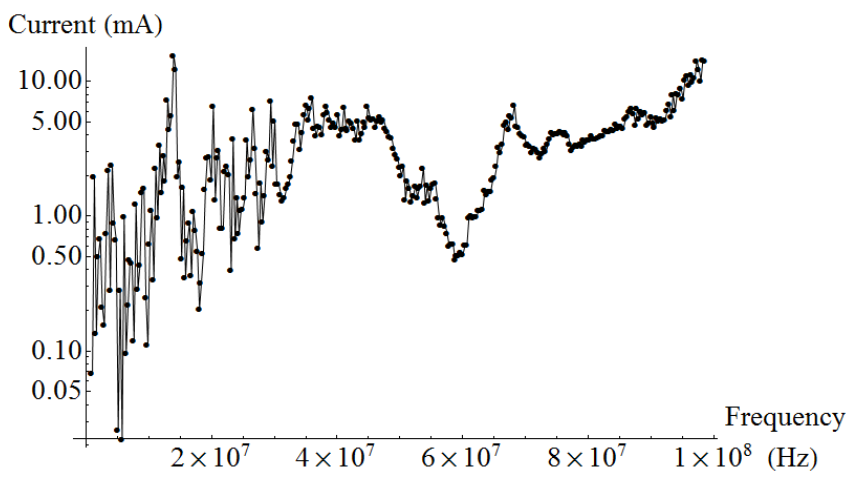

Figure 8: Mean level of the mean magnitude $\left(a_{0, \mu}\right)$ of endcurrent $\left|\mathrm{I}_{\text {out }}\right|$

It can be observed that the effect induced by the position of conductors in the electrical raceway (Fig. 9) and also the effect induced by connected appliances (Fig. 10) are related to the frequency.

It is interesting to note that depending on the frequency, the mean effects $a_{1, \mu}$ and $a_{2, \mu}$ can be positive or negative which correspond respectively to the decrease and the increase of the spurious mean end-current. 


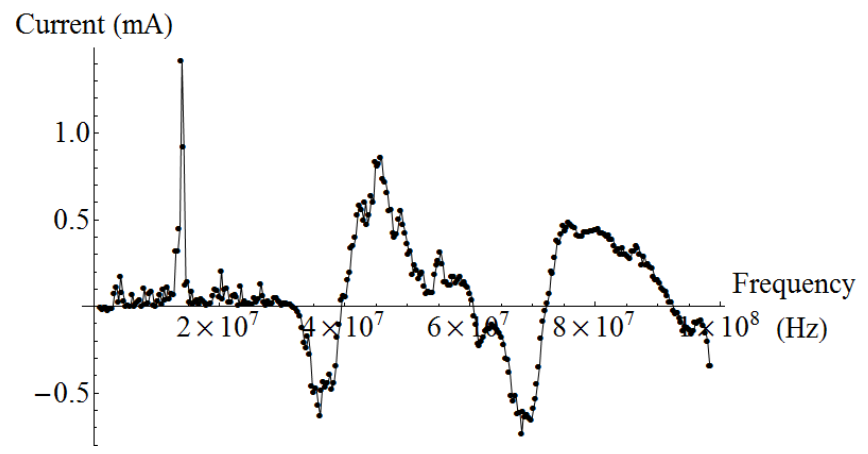

Figure 9: Effects induced by random positions of conductors in the electrical raceways (factor 1) on the mean end-current magnitude $\left(a_{1, \mu}\right)$

Moreover, as it is depicted in Fig. 9 and Fig. 10, the highest effects are reached for the following frequencies: $25 \mathrm{MHz}$, $50 \mathrm{MHz}$ and $75 \mathrm{MHz}$ (their respective wavelength $\lambda=12 \mathrm{~m}$, $6 \mathrm{~m}$ and $4 \mathrm{~m}$ ).

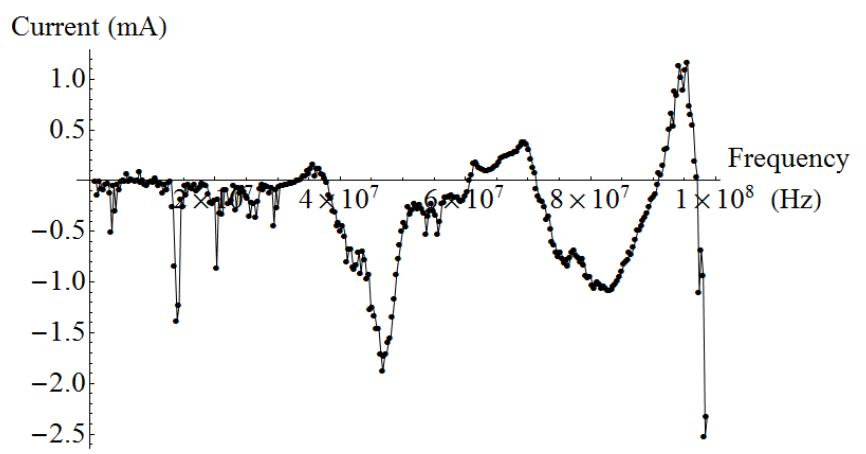

Figure 10: Effects induced by connected appliances (factor $2)$ in the low voltage network on the mean end-current magnitude $\left(a_{2, \mu}\right)$

This can be explained by the fact that, for some values of frequency, the characteristic impedance matrices of the cable and of the appliances are matching; it can be pointed out that resonances also occur. Same conclusions may be drawn about the interaction between factors.

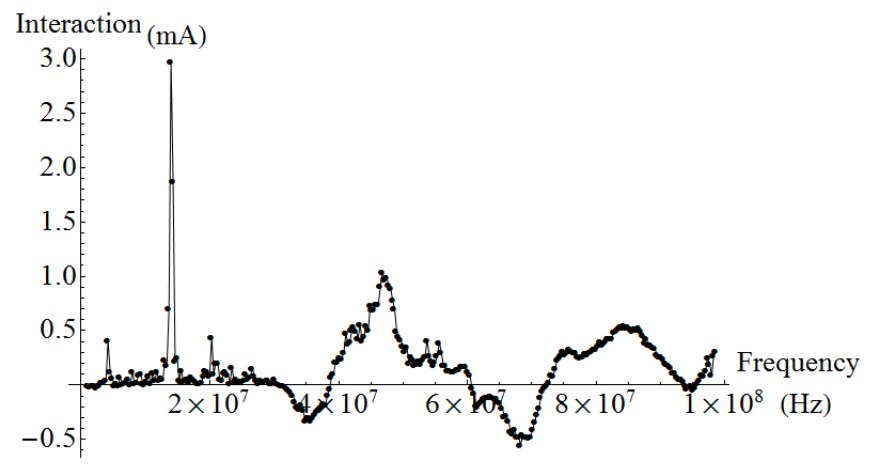

Figure 11: Interactions between both factors $\left(a_{12, \mu}\right)$

But it is difficult to give further conclusions looking just at the variations of the effects with regards to the frequency. Therefore, a statistical study has been performed assuming that the frequency behaves now as a random variable. The aim is still to hierarchise the influence of the considered factors.

\subsubsection{Considering the frequency as a random variable}

In order to estimate the general effects of the input modelling parameters in the working frequency band, we propose an analysis of the statistical moments of the probability density functions (PDFs) of the computed effects. Such a study is justified since we would like to estimate the influence of each factor in order to try to reduce the propagation of spurious compromising electromagnetic emanations whose frequency is supposed to be unknown.

In Table 1 , the mean $\mu\left(a_{i}\right)$ and the standard deviation $\sigma\left(a_{i}\right)$ of the effects deduced from the Experimental Design are given. This will allow to hierarchise the effects and interaction PDFs. It can be pointed out that the mean level of the end-current mean magnitude $\mu\left(a_{l, u}\right)$ which is about $3.45 \mathrm{~mA}$, has a standard deviation of $2.68 \mathrm{~mA}$ in the defined working frequency band.

Table 1: Statistical moments of the Experimental Design applied to the mean end-current magnitude $\left|I_{\text {out }}\right|$ and to its standard deviation with factors 1 and 2

\begin{tabular}{ccc}
$\begin{array}{c}\text { Parameter } \\
\text { under study }\end{array}$ & $\begin{array}{c}\boldsymbol{\mu}\left(\boldsymbol{a}_{i}\right) \\
\boldsymbol{m} \boldsymbol{A}\end{array}$ & $\begin{array}{c}\sigma\left(\boldsymbol{a}_{i}\right) \\
\boldsymbol{m} \boldsymbol{A}\end{array}$ \\
\hline $\boldsymbol{a}_{\boldsymbol{0}, \boldsymbol{\mu}}$ & 3.45 & 2.68 \\
$\boldsymbol{a}_{\boldsymbol{0}, \boldsymbol{\sigma}}$ & 1.21 & 1.21 \\
$\boldsymbol{a}_{1, \boldsymbol{\mu}}$ & 0.09 & 0.30 \\
$\boldsymbol{a}_{\boldsymbol{1 , \sigma}}$ & 0.21 & 0.44 \\
$\boldsymbol{a}_{2, \boldsymbol{\mu}}$ & -0.28 & 0.53 \\
$\boldsymbol{a}_{2, \sigma}$ & -0.17 & 0.70 \\
\hline
\end{tabular}

First the standard deviation $\sigma\left(a_{0, \mu}\right)$ of the average of the mean effect is high compared to the mean $\mu\left(a_{0, \mu}\right)$. This points out the dispersion of the parameter $a_{0, \mu}$ due to the frequency as it has been observed in Fig. 8 and later confirmed by the significant mean of the standard deviation $\mu\left(a_{0,}\right)$.

Comparing $\mu\left(a_{1, \mu}\right)$ and $\mu\left(a_{2, \mu}\right)$, we may conclude that factor 2 (appliances) has a stronger influence on the observable $a_{0, \mu}$ than factor 1 (randomness of the cables). From the EMC point of view, it is more difficult to exploit the results obtained concerning higher statistical moments as $\sigma\left(a_{2,0}\right)$.

As it would have been expected, based on the retrieved impedances of appliances [17], the connected loads have a predominant effect on the conducted propagation of spurious compromising emanations. It has been shown, thanks to the study of the common-mode end-current, that the interleaving of cables has a little effect on the spurious end-current magnitude. 


\section{Conclusions}

The paper deals with the effects induced by several parameters on the conducted propagation of a spurious compromising electromagnetic interference in a realistic low voltage network. We have investigated both the effects induced by the randomness of conductor positions in the electrical raceway, and by the connected appliances thanks to the Generalized Experimental Design. It has been shown that due to the variability of the input modelling parameters, the prediction of spurious end-current magnitude remains very difficult.

Nevertheless, it has been pointed out that the appliances have a dominant effect on the conducted propagation of spurious compromising emanations. Thus, we can conclude that the estimation of conducted propagation of spurious compromising emanations should be performed on a network loaded by general appliances and not left unloaded.

In supplement, the Design of Experiments methodology should be performed on the differential-mode end voltages and currents in order to estimate the effects induced by these parameters on the conducted propagation of electromagnetic waves in commercial building. It will be finally possible to compare both study and also to highlight the effects of both parameters.

\section{Acknowledgements}

This work is supported by the French Network and Information Security Agency (ANSSI); it emanates from a bilateral collaboration between University Pierre and Marie Curie (L2E) and ANSSI.

\section{References}

[1] G. H. Baker and C. J. Elliott, "Cascading Infrastructure Failures, Avoidance and Response", Harrisonburg, VA Institute for Infrastructure and Information Assurance", James Madison University, 2008.

[2] R. L. Gardner and D. C. Stoudt, "Extension of the hybrid modeling technique to the susceptibility of complex infrastructure circuits and systems", Electromagnetics in Advanced Applications (ICEAA), 2011 International Conference on , vol., no., pp.773,776, 12-16 Sept. 2011.

[3] D. Mansson, R. Thottappillil, and M. Backstrom, "Propagation of UWB Transients in Low-Voltage Power Installation Networks," Electromagnetic Compatibility, IEEE Transactions on, vol. 50, pp. 619629, 2008.

[4] D. Mansson, T. Nilsson, R. Thottappillil, and M. Backstrom, "Propagation of UWB Transients in LowVoltage Installation Power Cables," Electromagnetic Compatibility, IEEE Transactions on, vol. 49, pp. 585592, 2007.

[5] F. Rachidi, C.A. Nucci, M. Ianoz and C. Mazzetti, "Response of multiconductor power lines to nearby lightning return stroke electromagnetic fields", IEEE
Transactions on Power Delivery, Vol. 12, pp. 14041411, 1997.

[6] F. M. Tesche, M. V. Ianoz and T. Karlsson, "EMC Analysis Methods and Computational Models", Wiley, New York, 1999.

[7] C. Kasmi, M. Hélier and M. Darces, "Statistical analysis of a spurious signal level in a low voltage PLC network”, EMC Symposium 2012, Rome, 2012.

[8] D. V. Giri and F. M. Tesche, "Classification of Intentional Electromagnetics Environments (IEME)", IEEE Transactions on Electromagnetic Compatibility, Vol. 4, pp. 322-328, August 2004.

[9] F. Sabath, "Threat of Electromagnetic Terrorism: lessons learned from documented IEMI Attacks", EUROEM 2012, Toulouse, France, July 2012.

[10] M. Vuagnoux, "An improved technique to discover compromising electromagnetic emanations", EMC symposium, Fort Lauderdale, USA, August 2010.

[11]D. C. Montgomery, Design and Analysis of Experiments, ISBN-13 978-1118146927, Wiley, 2012.

[12] C.R. Paul, "Analysis of Multiconductor Transmission Lines”, John Wiley \& Sons, New-York, 1994.

[13] J-P. Parmantier and S. Bertuol, "CRIPTE Training and Electromagnetic coupling on cable networks", ONERA, Toulouse, France, 2011.

[14] J-P. Parmantier, P. Degauque, "Topology Based Modeling of Very Large Systems". Modern Radio Science 1996, pp. 151-177, edited by Hamelin. Oxford University Press.

[15] C. J. Kim and M. Chouika, "Attenuation Characteristics of High Rate Home-Networking PLC Signals", Transaction on power delivery, Vol. 17, NO. 4, October 2002.

[16] D. Hirata, Y. Akiyama, H. Yamane, "Influence of Appliance State on Transmission Characteristics of Indoor AC Main Lines in Frequency Range Used Power Line Communication", 2005.

[17] C. Kasmi, M. Hélier, M. Darces, "HF impedance measurement of electronic devices using a deembedding technique", in Proc. of EUROEM Conf., Toulouse, July 2012.

[18] J.-P. Parmantier, "An efficient technique to calculate ideal junction scattering parameters in multiconductor transmission line networks", Note 536, February 24, 1998.

[19] R. A.Fisher, "The Design of Experiments", $9^{\text {th }}$ edition, Macmillan. ISBN 0-02-844690-9, 1971. 